\title{
Twinning and stacking fault formation during tensile deformation of nanocrystalline $\mathrm{Ni}$
}

\author{
X. Wu ${ }^{\text {a,* }}$, Y.T. Zhu ${ }^{\text {b }}$, M.W. Chen ${ }^{\text {c }}$, E. Ma ${ }^{\text {d,* }}$ \\ ${ }^{a}$ State Key Laboratory of Nonlinear Mechanics, Institute of Mechanics, Chinese Academy of Sciences, Beijing 100080, China \\ ${ }^{\mathrm{b}}$ Los Alamos National Laboratory, Los Alamos, NM 87545, USA \\ ${ }^{c}$ Institute of Materials Research, Tohoku University, Sendai, Japan \\ ${ }^{\mathrm{d}}$ Department of Materials Science and Engineering, Johns Hopkins University, Baltimore, MD 21218, USA
}

Received 14 November 2005; received in revised form 20 December 2005; accepted 26 December 2005

Available online 13 February 2006

\begin{abstract}
Deformation twins and stacking faults have been observed in nanocrystalline $\mathrm{Ni}$, for the first time under uniaxial tensile test conditions. These partial dislocation mediated deformation mechanisms are enhanced at cryogenic test temperatures. Our observations highlight the effects of deformation conditions, temperature in particular, on deformation mechanisms in nanograins.
\end{abstract}

(C) 2006 Acta Materialia Inc. Published by Elsevier Ltd. All rights reserved.

Keywords: Nanostructure; Twinning; Plastic deformation; Nickel

\section{Introduction}

The plastic deformation mechanisms of nanocrystalline (NC) metals have been under intense investigation in recent years. The formation of deformation twins and stacking faults, indicating the presence of dislocation activities in $\mathrm{NC}$ grains and especially the operation of partial dislocation mediated processes (PDMPs), has been considered as a contributing deformation mechanism [1-7], based on recent transmission electron microscopy (TEM) observations in $\mathrm{Al}$ [1-3], $\mathrm{Cu}$ [4], Pd [5,6] and Ta [7]. So far, all of the experimental evidence for PDMPs have been obtained in NC metals that were subjected to complicated stress states and high stress levels, such as in indentation [1], grinding [1], high-pressure torsion [4], high-rate cold rolling $[5,6]$, and ball milling (sometimes with powders immersed in liquid nitrogen $[2,3])$. It is not clear if the PDMPs would occur in a uniaxial tensile test, which arguably evaluates the most important mechanical properties of a material.

\footnotetext{
* Corresponding authors.

E-mail addresses: xlwu@imech.ac.cn (X. Wu), ema@jhu.edu (E. Ma).
}

It is believed that the PDMPs, if operative, could significantly affect the tensile behavior of NC metals [2-4]. Therefore, it is of significant interest to investigate if the PDMPs are activated during a tensile test.

In a recent room temperature (RT) tensile test [8], in situ $\mathrm{X}$-ray diffraction studies of $\mathrm{NC} \mathrm{Ni}$ subjected to tensile deformation showed no irreversible peak broadening, indicating that no twin or stacking faults were formed. This result raises important questions. First, would PDMPs ever occur in $\mathrm{NC} \mathrm{Ni} \mathrm{during} \mathrm{conventional} \mathrm{tensile/compression}$ tests? Second, if PDMPs do get activated in NC Ni grains under the stress state of a uniaxial test, what are the deformation parameters (such as temperature and strain rate) that make them happen, and how could the absence of PDMPs in the RT tensile test of Ref. [8] be explained?

We provide answers to these questions by extensive TEM observations in $\mathrm{NC} \mathrm{Ni}$ subjected to tensile tests at different temperatures. This is therefore also the first observation of the dependence of deformation mechanisms not only on the nanocrystalline grain size but also on deformation conditions (such as temperature). Such a study is especially valuable in the attempt to construct deformation maps for nanocrystalline materials [9], which requires a 
complete set of information regarding the effects of grain size, temperature, and strain rate. We demonstrate that PDMPs do occur in NC Ni under uniaxial tensile deformation. But the activation of PDMPs was frequently observed only in tensile tests at cryogenic temperatures, and correspondingly at a flow stress level higher than that at RT. The results will be discussed in light of the predictions by molecular dynamics (MD) simulations [9-11] and analytical models $[1,12,13]$ for NC face-centered cubic metals.

\section{Experimental}

An electrodeposited $\mathrm{NC} \mathrm{Ni}$ foil was acquired from Goodfellow Inc. The as-received $150 \mu \mathrm{m}$ thick foil was observed to have an average grain size of $\sim 25 \mathrm{~nm}$, similar to those reported before [14-17]. Dog-bone shaped tensile samples were prepared using the electrical discharge technique, to the gauge length of $8 \mathrm{~mm}$ and width of $4 \mathrm{~mm}$. The samples were tested under tension to failure at a strain rate of $3 \times 10^{-3} \mathrm{~s}^{-1}$, either at RT, or at liquid nitrogen temperature (LNT) by immersing the tensile sample and grips in liquid nitrogen. The tensile behavior at these temperatures was similar to those published before for electrodeposited $\mathrm{NC} \mathrm{Ni}$ [14-17]. The strain sustained before failure is approximately $4 \%$, at which point the flow stress (the maximum stress on the respective stress-strain curves) is about $1200 \mathrm{MPa}$ at RT and $1500 \mathrm{MPa}$ at LNT [16]. The microstructures were examined using a high-resolution transmission electron microscope (HRTEM), JEM2010F operated at $200 \mathrm{kV}$. TEM samples after tensile tests were prepared as follows. The fractured tensile specimens were mechanically thinned down to about $50 \mu \mathrm{m}$ thick. $2 \mathrm{~mm}$ long and $1 \mathrm{~mm}$ wide slices were then cut parallel to the tensile axis. Needle-shaped specimens glued to a metallic ring were produced via electropolishing, followed by Ar ion milling using a Gatan Precision Ion Polishing System with a small incident angle, allowing large, thin and homogeneous areas to be obtained at the tip and edge areas. A TEM specimen is shown in the scanning electron micrograph of Fig. 1.

\section{Results}

We first examined several as-received $\mathrm{NC} \mathrm{Ni}$ samples. Pre-existing growth twins were few and far between in the $\sim 100$ grains we examined, consistent with the TEM observations in Ref. [14]. We also examined samples pulled to failure after the RT tensile tests. Again we only observed very few twins (no more than 5 in $\sim 120$ grains) and almost no full dislocations in the NC grains. Some of the twins observed may be growth twins. This finding is consistent with the published X-ray peak broadening results [8]. The comparison of grain size distribution before and after tensile tests is presented in Fig. 2. The mean grain sizes are $23.8 \pm 6.2 \mathrm{~nm}$ and $24.6 \pm 8.1 \mathrm{~nm}$, and the peak values are $18.6 \mathrm{~nm}$ and $19.0 \mathrm{~nm}$, respectively. The changes are very small after the $3-4 \%$ straining in the tensile tests [16].

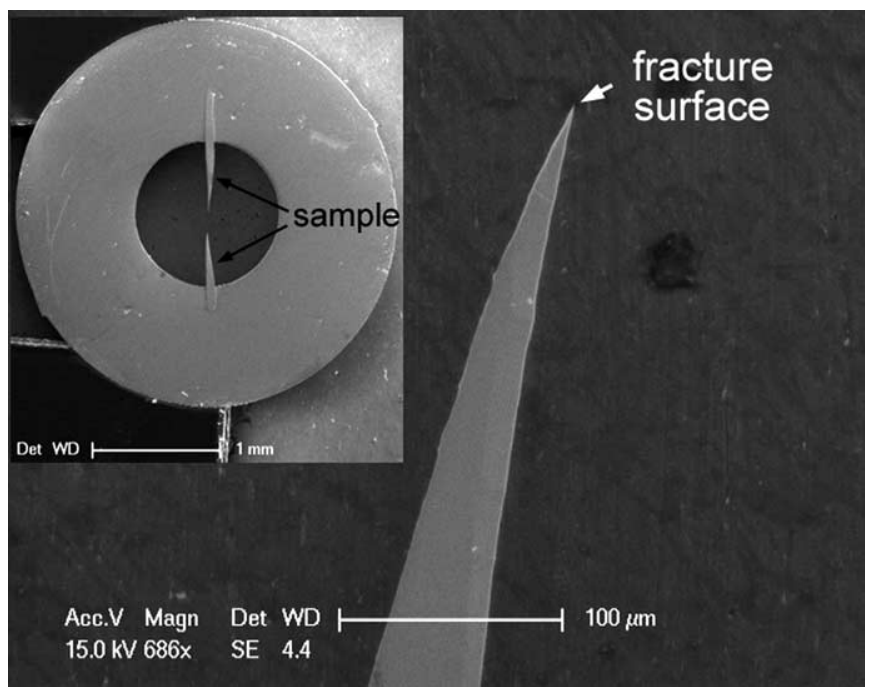

Fig. 1. Scanning electron micrograph of TEM specimens made from the tensile sample. The tip and two sides are areas for observation. The inset shows two specimens bonded to a TEM sample ring.
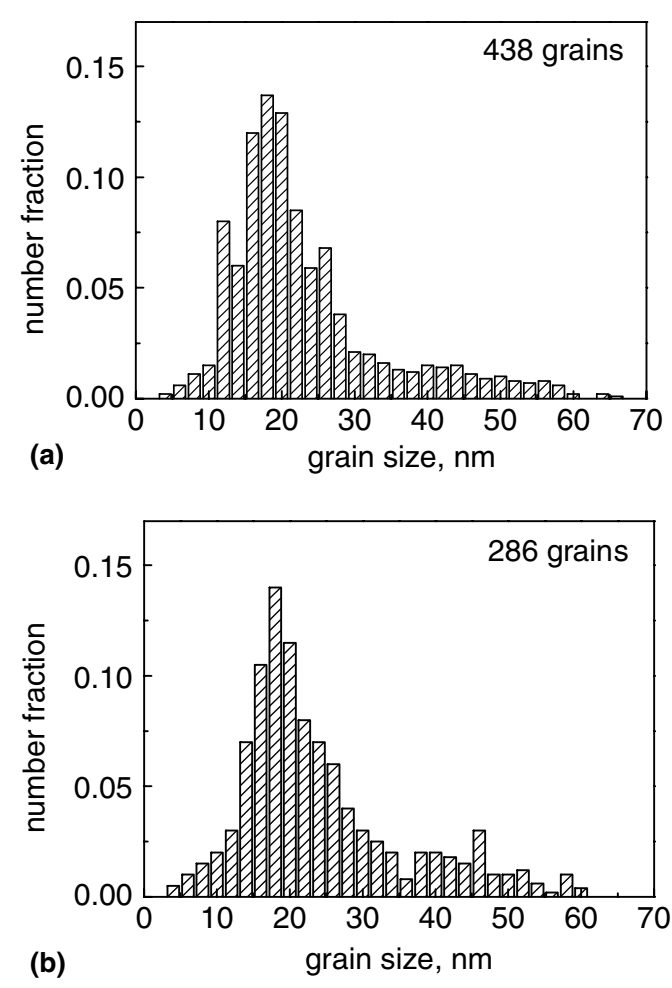

Fig. 2. Grain size distribution (a) prior to and (b) after LNT tensile deformation, obtained using TEM bright-field pictures.

In contrast, after the LNT tests deformation debris was found readily in TEM observations. Fig. 3(a) is a brightfield TEM image showing the microstructure near the fracture surface. Grain A, with a diameter of about $25 \mathrm{~nm}$, is an example. An enlarged view of this grain, Fig. 3(b), clearly reveals mechanical twins. Two twin boundaries are indicated by arrows, and the twin relations are marked in the HRTEM image of Fig. 3(c). Such twins have been 

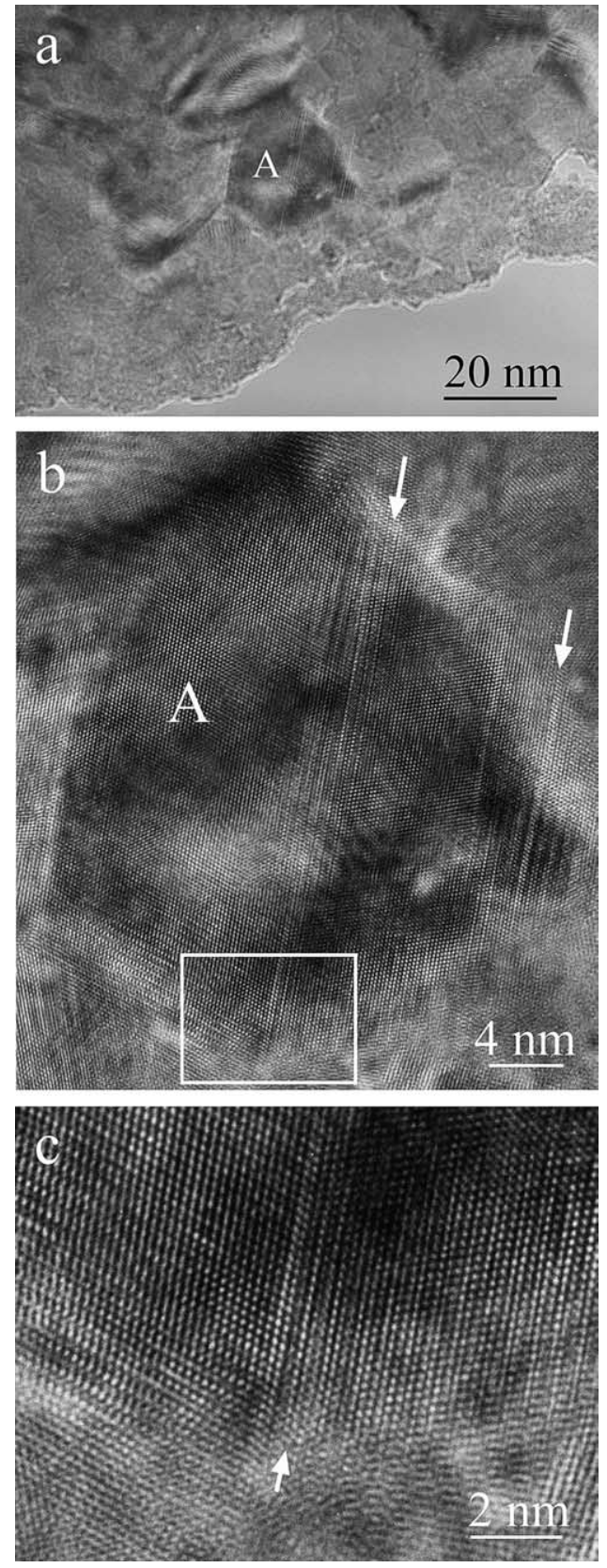

Fig. 3. (a) A low-magnification image of $\mathrm{NC}$ grains near the tip of the fracture surface. Note an equi-axed NC grain labeled A. (b) HRTEM image of grain A. (c) Details of the twin boundary (arrow) in (b).

observed in a number of nanograins. Fig. 4(a) shows a nanograin in which many stacking faults have also been observed in addition to deformation twins. In fact, high densities of stacking faults are often seen, another example of which is shown in Fig. 4(b). Some of these stacking faults and microtwins do not pass across the whole grain, but stop in the grain interior with Shockley partial dislocations located at the front boundaries of the stacking faults and microtwins. This indicates that these twins heterogeneously nucleated at the grain boundary (GB) and grew into the grain interior via partial dislocation emission from the GB. We examined many locations along the edge of the

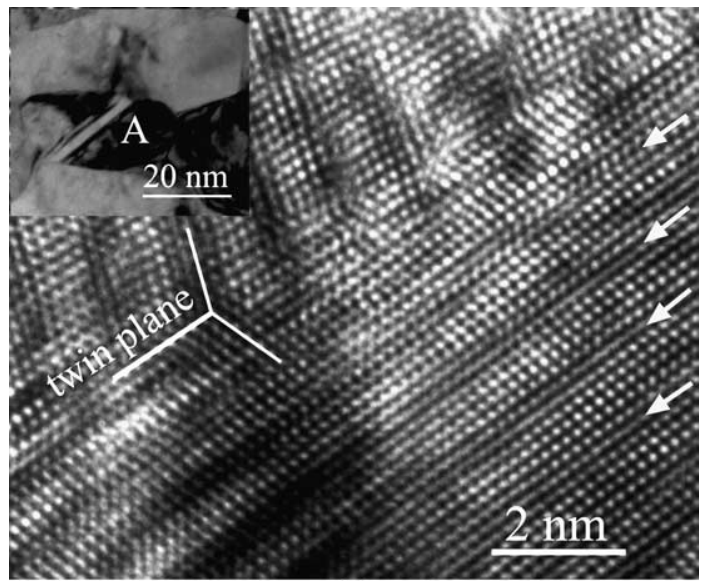

(a)

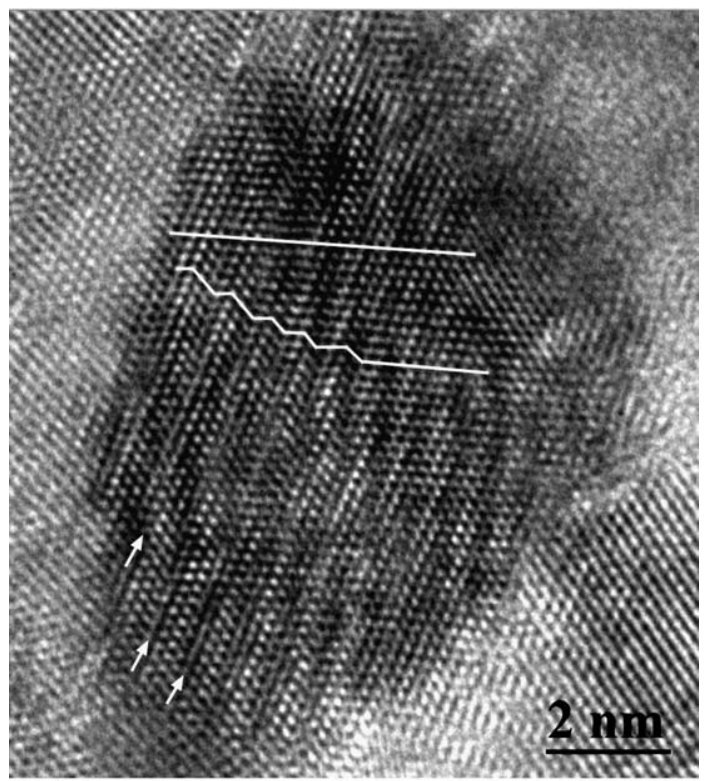

(b)

Fig. 4. (a) HRTEM micrograph of an NC grain in [011] orientation revealing a deformation twin as indicated by white lines. The arrows indicate stacking faults. A low magnification view of $\mathrm{NC}$ grain $\mathrm{A}$ is shown in the inset. (b) A typical [0 1 1 1] HRTEM image of an NC grain showing a number of microtwins and stacking faults with one end of the microtwins and faults ending inside the grain. The arrows indicate the formation of stacking faults originating from the grain boundary.

TEM specimen, intentionally at different distances from the tip (the tip is closer to the fractured region where the stress state may be complex; no necking was observed in our tensile samples). We also checked eight TEM specimens from several tensile samples. In all cases, we observed PDMP features similar to those presented in Figs. 3 and 4. Therefore, we conclude that deformation twins and stacking faults are a common occurrence during the low temperature tensile deformation of NC Ni. Some full dislocations were found as well, as discussed below.

Prior investigations of deformation twins observed only co-planar twins (twin planes parallel to one another) in a given $\mathrm{NC}$ grain [1,5], i.e., only one twinning system is activated in a particular grain. We observed, occasionally, two 


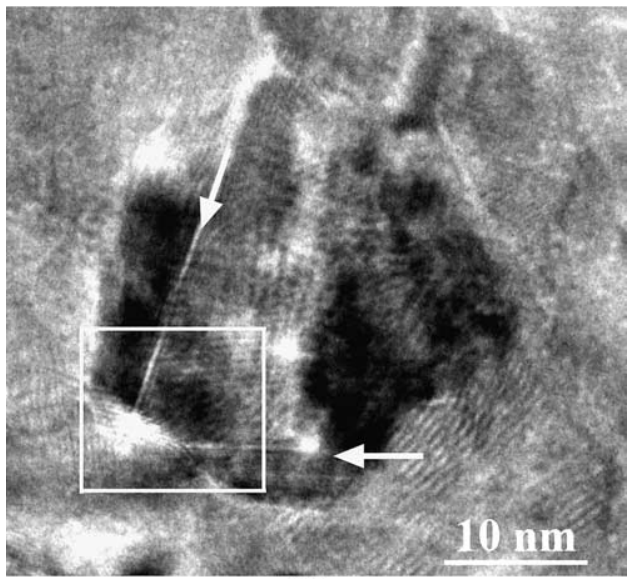

(a)

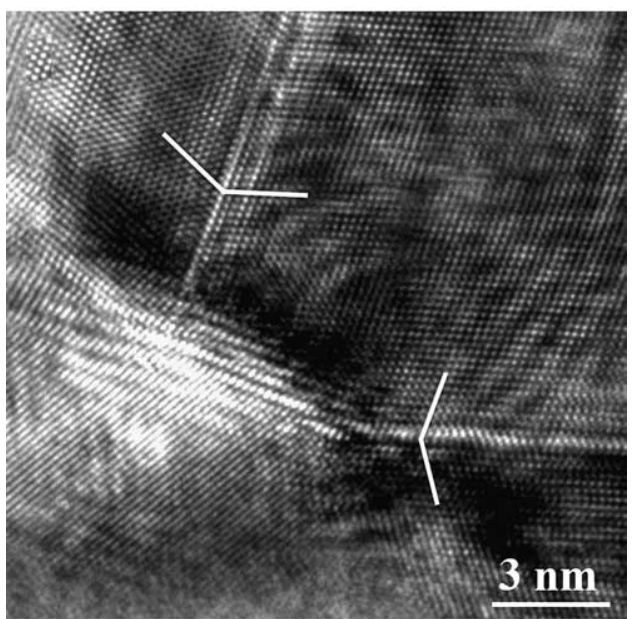

(b)

Fig. 5. (a) A low-magnification micrograph of an equi-axed $\mathrm{NC}$ grain. The twin boundaries of two different orientations are indicated by arrows. (b) HRTEM micrograph of the twins.

twinning systems in operation in a single grain. This scenario is shown in Fig. 5, where the two sets of deformation twins are marked with arrows.

\section{Discussion}

Our experiments above clearly indicate that PDMPs, i.e., twinning and stacking fault formation, not only occurred in $\mathrm{NC} \mathrm{Ni}$, but also contributed to tensile deformation under the uniaxial stress state. The only previous observation of twinning during tensile deformation of an $\mathrm{NC}$ metal was made on an electrodeposited $\mathrm{Ni}$ foil in situ deformed inside a TEM at RT [15]. Signs of deformation twinning were found in the last ligament before fracture. However, in such a TEM foil most of the deformation is associated with the advancement of a crack tip where unusual deformation mechanisms may be operating. Meanwhile, when only a few grains are sitting on top of each other near the free surfaces, dislocation activities, diffusional processes, and changes in the GB structures may have been enhanced. Therefore, it is questionable if the in situ observations in thin TEM foils are relevant to bulk deformation. We found that the RT tensile test was not a condition conducive to PDMP activation, but LNT significantly promoted PDMP operation, even though the overall plastic strain was not large. It is well known for conventional metals/alloys that a low-deformation temperature (or high strain rate) favors twinning [18]. This is usually explained as a consequence of the suppression of the thermal activation of normal dislocations, raising the applied stresses. The same is apparently true also for NC metals. In the following, we discuss further why PDMPs are much more favored at LNT than RT, and compare our findings with prior modeling results.

For NC Ni at RT, MD simulations [19] predicted that dislocation sources are located at GBs only, and the dislocations are emitted in the form of partial dislocations one at a time. The leading partial travels across the entire grain with no following trailing partial, leaving behind a stacking fault defect [19]. Explanations have been given before that under high stress conditions there can indeed be such a preference for "one partial at a time" [1]. But the high flow stresses in MD simulations come from the unrealistically high strain rates not accessible in laboratory experiments. In the RT tensile tests the stress level is approximately 1-1.2 GPa which, according to our analytical model estimates based on the equations in Ref. [18], is only sufficient to nucleate full dislocations from the GBs. In fact, dissociated full dislocations require lower stress to nucleate in $>27 \mathrm{~nm} \mathrm{Ni}$ grains than twinning dislocations [18]. Once nucleated, the extended dislocation in NC Ni at RT has short splitting distances and behaves as a normal perfect dislocation.

In addition, if in some local GB regions the stress was high enough to emit partials, at RT the trailing partial is more likely to come out than at LNT. The trailing partial erases the stacking fault as it runs across the grain to reach the opposite grain boundary, leaving no footprint for detection in post-mortem TEM (ours and others [15]) or X-ray peak broadening [8] studies. It was not possible to accomplish this during the very limited time available in an MD run [19], because there is a waiting period needed for the stress build-up and for the atomic shuffling/ mobility/rearrangements in the GB to nucleate the trailing partial, especially since the latter has a Burgers vector different from the leading partial. But, this is not a problem on the laboratory time scale, with the thermal energy available at RT. On the other hand, our infrequent observations of PDMPs suggest that they are not a main contributor during RT tensile deformation. Otherwise we would observe some stacking faults. This is because if a grain is oriented in such a way that the GB emission of the first partial is favored (e.g., the shear stress is parallel to its Burgers vector $\mathbf{b}_{1}$ ), it would be much more difficult to emit the trailing partial because it would have a $\mathbf{b}_{2}$ in an unfavorable orientation. As a result, statistically it is not likely that all the stacking faults left by the leading partials would be erased by the trailing partials.

Now let us consider what may happen when the deformation is carried out at LNT. The dislocation processes 
are thermally activated, especially in $\mathrm{NC}$ metals that show a stronger-than-normal temperature/strain rate dependence of the flow stresses $[16,20,21]$. In contrast to RT, now the relaxation/adjustment in the grains and GBs is depressed, and the activation of dislocations would then rely largely on the local stress intensity (depending on the applied stresses, internal stresses, and the stress field of the leading partial) alone, with much less help from thermal fluctuation. Consequently, the deformation flow stress observed in a LNT tensile test is higher than that at RT, and there can be large variations of local stresses, at least more nonuniform than the RT case. These allow the various PDMPs to become operative. The higher strength at the low temperature produces a larger difference in the resolved shear stress between the leading and trailing partials with different Schmid factors, which will reduce the probability of the trailing partial and favor twin nucleation that requires partials with the same Burgers vector as for the leading partial. Thus, twinning may become kinetically competitive at the low temperature. When the trailing partial is not forced out due to insufficient local stress intensity and unfavorable Schmid factor, a stacking fault is left.

The third defect configuration is the full dislocations seen in Fig. 6. These may be emitted as full dislocations from the GBs, or formed when the trailing partial catches up with the leading. Note that the static energy barriers, given by the planar fault energy curves as depicted in MD simulations [11], are not very different in $\mathrm{Ni}$ for the several partial dislocation processes. When the deforma-

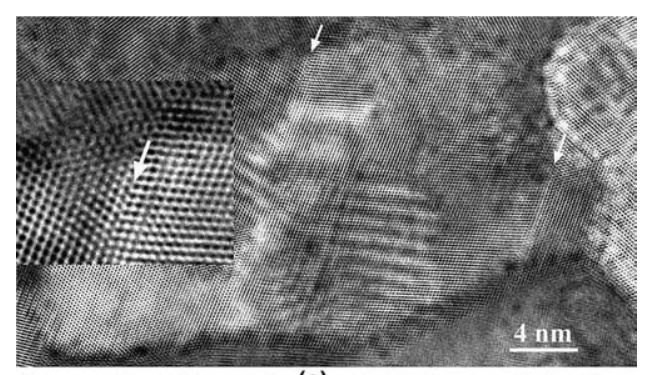

(a)

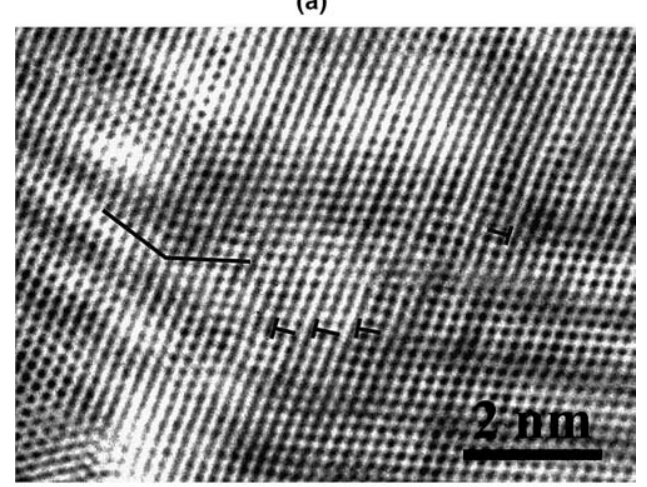

(b)

Fig. 6. (a) A low-magnification TEM micrograph of an NC grain. Two parallel twin boundaries are indicated by arrows. The inset shows the details of twin boundary of the left side. (b) HRTEM image of full dislocations trapped inside the nanograin not far from the twin boundary. tion stress is forced to reach high levels, all the PDMPs are expected [11]. As a result, stacking faults (due to the absence of the trailing partial), full dislocations (with trailing partial having a different Burgers vector, on the same plane), and deformation twins (partial with the same Burgers vector but on consecutive planes) are all competitive alternatives. Trapped full dislocations are not often observed post mortem, in RT deformed samples [14,15,22]. Their population becomes considerable after our cryogenic test, as the reduced mobility renders pinning more likely, e.g., by GBs along which the two ends of the dislocation glide. The exact reason for the pinning (e.g., due to impurities) or locking (e.g., due to the formation of jogs) remains to be further explored.

\section{Summary remarks}

We have shown that PDMPs, including twinning and stacking fault formation, do get activated in NC Ni. This is the first demonstration for the partial dislocation mediated deformation mechanisms in an NC metal under uniaxial tensile conditions. Similar to the case for conventional grain sizes, twinning is promoted at low-deformation temperatures. Our explanations are based on the competing thermally activated processes mediated by full or partial dislocations. At cryogenic temperatures, PDMPs are favored due to the high deformation stresses. The kinetic/geometric constraints restrict the nucleation of the trailing partial, rendering the subsequent process largely dependent on local stress conditions. As a result, stacking faults, full dislocations and deformation twins are all competing alternatives that have been readily captured in post mortem TEM observations. Our results also indicate that defect storage in $\mathrm{NC}$ grains is significant during cryogenic deformation and deformation mechanisms are obviously temperature dependent. PDMPs would also be enhanced at high strain rates and/or large plastic strains, when high stresses are needed to keep up with the applied strain rate and the stress concentrations become large and varied.

\section{Acknowledgments}

XW was supported by the National Natural Science Foundation of China under grant Nos. 50471086, 50571110, 10472117, 50021101, the National 973 Program of China under grant No. 2004CB619305 and EM by US NSF-DMR-0210215 and DMR-0355395.

\section{References}

[1] Chen MW, Ma E, Hemker KJ, Sheng HW, Wang YM, Cheng X. Science 2003;300:1275.

[2] Liao XZ, Zhou F, Lavernia EJ, He DW, Zhu YT. Appl Phys Lett 2003;83:632.

[3] Liao XZ, Zhou F, Lavernia EJ, He DW, Zhu YT. Appl Phys Lett 2003;83:5062. 
[4] Liao XZ, Zhao YH, Srinivasan SG, Zhu YT, Valiev RZ, Gunderov DV. Appl Phys Lett 2004;84:592.

[5] Rosner H, Markmann J, Weissmuller J. Philos Mag Lett 2004;84:321.

[6] Markmann J, Bunzel P, Rösner H, Liu KW, Padmanabhan KA, Birringer R, et al. Scripta Mater 2003;49:637.

[7] Wang YM, Hodge AM, Biener J, Hamza AV, Barnes DE, Liu K, et al. Appl Phys Lett 2005;86:101915.

[8] Budrovic Z, Van Swygenhoven H, Derlet PM, Van Petegem S, Schmitt B. Science 2004;304:273.

[9] Yamakov V, Wolf D, Phillpot SR, Mukherjee AK, Gleiter H. Nature Mater 2004;3:43.

[10] Yamakov V, Wolf D, Phillpot SR, Gleiter H. Acta Mater 2002;50: 5005.

[11] Van Swygenhoven H, Derlet PM, Frøseth AG. Nature Mater 2004; 3:399.

[12] Asaro RJ, Krysl P, Kad B. Philos Mag Lett 2003;83:733.
[13] Asaro RJ, Suresh S. Acta Mater 2005;53:3369.

[14] Dalla Forre F, Van Swygenhoven H, Victoria M. Acta Mater 2002; 50:3957.

[15] Kumar KS, Suresh S, Chisholm MF, Horton JA, Wang P. Acta Mater 2003;51:387.

[16] Wang YM, Ma E. Appl Phys Lett 2004;85:2750; Wang YM, Hamza A, Ma E. Appl Phys Lett 2005;86:241917.

[17] Wang YM, Cheng S, Wei QM, Ma E, Nieh TG, Hamza A. Scripta Mater 2004;51:1023.

[18] Zhu YT, Liao XZ, Srinivasan SG, Lavernia EJ. J Appl Phys 2005;98: 034319.

[19] Van Swygenhoven H. Mater Sci Forum 2006;503-504:193.

[20] Wang YM, Ma E. Appl Phys Lett 2003;83:3165.

[21] Wei Q, Cheng S, Ramesh KT, Ma E. Mater Sci Eng A 2004;381:71.

[22] Hugo RC, Kung H, Weertman JR, Mitra R, Knapp JA, Follstaedt DM. Acta Mater 2003;51:1937. 